\title{
Recursive Identification Based on OS-ELM for Emotion Recognition and Prediction of Difficulties in Video Games
}

\author{
Hayfa BLAIECH ${ }^{1 *}$, Noureddine LIOUANE ${ }^{2}$ \\ ${ }^{1}$ National Engineering School of Monastir ENIM, Laboratory of Automatic, \\ Signal and Image Processing (LARATSI), Tunisia \\ hayfaelblaiech@gmail.com (*Corresponding author) \\ ${ }^{2}$ National Engineering School of Monastir ENIM, University of Monastir, Tunisia \\ noureddine.liouane@enim.rnu.tn
}

\begin{abstract}
The aim of this paper is to recognize human emotions from physiological electroencephalography (EEG) signal. Indeed, this paper demonstrates that neural networks in conjunction with recursive least squares can be used sprucely for the identification of emotional states. In this regards, this report uses online sequential extreme learning machine to conceive the present emotion recognition system. To validate the efficiency of this machine learning, the performance of various popular feature extraction methods with the Database for Emotion Analysis using Physiological Signals DEAP dataset, and two newly developed EEG datasets are systematically evaluated for this study. The first emotional experience was to conceive an emotional dataset containing the samples EEG of six selected emotion states (neutrality, amusement, surprise, compassion, attraction, and disgust), and the second one was to predict the difficulties encountered during playing a hard game. This has the goal to detect the concentration and anger emotional states. The purpose of this paper is to improve the accuracy of emotion recognition based on different emotional states. The features are extracted from the delta, theta, alpha and beta bands. Based on the analysis of the identification system, the most significant features are extracted for each emotional state. The selected ones are then utilized in the emotion classification system. The present recognition system has $80 \%$ accuracy when using the DEAP database, $74.07 \%$ accuracy when using the first database, and obtains $61.11 \%$ accuracy when predicting difficulties in video games.
\end{abstract}

Keywords: Recursive identification, OS-ELM, Emotion Recognition, EEG signals, Video games, Feature extraction.

\section{Introduction}

Affective computing is one of the hottest topics in the field of human-computer interaction. It affects computer science, psychology and cognitive science. Affective computing, introduced by (Picard, 2000), seeks to give space to emotion in the field of computing (machines, robots, virtual agents). It is about giving a computer the ability of recognizing and interpreting personal emotions and affective behaviors, developing one's ability to respond intelligently to human emotion, helping in decision-making, and enhancing interaction and making it more effective and more natural. (Picard, 2000) split this affective computing into two research paths: the modeling of emotional processes in computational systems, and the design of systems allowing the automatic recognition of emotion. The first way is to generate virtual emotion in computational systems to improve communication with a human user. These computational systems can be virtual agents or expressive avatars. The second pathway discusses the recognition of emotion. It concerns how to use physiological and somatic indices to determine the user's emotion, utilizing visual and acoustic signal sensors. This recognition of the emotional state may play a major role in the system or improve the outcome of a system. Similarly, affective computing enhances the brain computer interaction systems with the ability of detecting, processing, and responding to the users' affective states utilizing physiological signals. To measure emotion, the three emotional components must be estimated: cognitive (self report of the subject using self-report instruments such as questionnaires and Self Assessment Manikin (SAM) (Barrett \& Russel, 1998), behavioral (facial expression like Facial Action Coding System (FACS) and Electromyography (EMG)), and physiological (cardiac rhythm, physiological signals, respiration, etc.). In this paper, the cognitive component will be inserted in the emotional experience done from EEG signals. The EEG signals will represent the physiological component. In this paper, the focus is on the emotion recognition from physiological EEG signals. The individual's variations in emotion as well as the different parameters of emotion, such as time, context, space and culture, make this issue very challenging. Moreover, human emotion is very complicated. In some cases, facial expressions do not give the exact emotion, which reveal the importance of analyzing EEG signals. The paper is written as follows. In section 2, some related works from the specialized literature are mentioned. In section 3, the machine learning 
based on Recursive Online Sequential Extreme Learning Machine (OS-ELM) is presented, and a novel algorithm based on the combination between recursive identification and OS-ELM is proposed. In section 4 , the experimental process followed for the construction of the two novel EEG databases based on discrete emotion and for the prediction of difficulties in video games is described. In section 5, the steps of the conception of the emotional recognition system formed by a recursive identification system and a classification system are presented. The results of the DEAP database and our two new databases are discussed and compared with other studies in section 6 . Finally, a conclusion of the present work is given in section 7 .

\section{Related Work}

In this section, the related work on emotion recognition systems based on EEG signals and other modalities is reviewed. Emotion recognition systems use two types of emotion models. Some studies have utilized the discrete model, yet others have used the valence arousal model. From the new works in facial expression recognition systems the authors (Yang et. al, 2018) can be cited as they presented the results of recognition of seven emotional states (neutral, joy, sadness, surprise, anger, fear, disgust) based on facial expressions. They used the coefficients describing elements of facial expressions, registered for six subjects, as features. The features have been calculated for three dimensional face model. The classification of features was performed using k-nearest neighbors (KNN) classifier and multilayer perceptron (MLP) neural network. They achieved $96 \%$ accuracy in the classification of emotions for random division of data and $73 \%$ accuracy in the classification for "natural" division of data. Also the authors in (Palaniswamy et al., 2018) proposed a technique for emotion recognition from facial expressions in images with simultaneous pose, illumination and age variation in real time. They considered the emotions anger, disgust, happy, surprise, and neutrality. The feature vectors that were formed from images from the CMU-MultiPIE database for pose and illumination were used for training the classifier. For real-time implementation, Raspberry Pi II was used, which can be placed on a robot to recognize emotions in interactive real-time applications. The proposed method includes face detection using Viola Jones Haar cascade, Active Shape Model (ASM) for feature extraction, and AdaBoost for classification in real-time. They obtained $96 \%$. Another example is the work of ( $\mathrm{Ng}$ et al. 2015), which classifies the emotions expressed by the primary human subject in static images extracted from movies. They follow a transfer learning approach for deep Convolutional Neural Network (CNN) architectures. They obtained an overall accuracy of $48.5 \%$ in the validation set and $55.6 \%$ in the test set. Concerning features extraction methods, the deep learning has recently become a hot research topic and has achieved state-of-the-art performance for a variety of applications ( $\mathrm{Li} \&$ Deng, 2018). Deep learning have been made in order to capture high-level abstractions through hierarchical architectures of multiple nonlinear transformations and representations. Up to now emotion recognition systems have had lack of performance in developing real-world application systems. To compare the present results to the ones of other works, only three publicly available emotion EEG datasets can be found. The public datasets are MAHNOB HCI (Balconi et al., 2015), DEAP (Koelstra et al., 2012) and SEED (Petrantonakis et al., 2009). In this study, an emotion recognition system based on several recursive identification subsystems is conceived, and the DEAP dataset is chosen to validate the effectiveness of our results.

\section{ELM and OS-ELM}

\subsection{ELM}

ELM machine is a type of neural network that guarantees the automatic learning of a linear model.

The ELM method is used for tracking the target of this paper. ELM can provide the automatic learning of a linear model. It is characterized by a single layer of hidden nodes, where the weights of hidden nodes connected to the inputs are randomly distributed and are always fixed. These machines have shown good generalization performance, robustness and controllability as well as a learning process which is much faster than the networks formed using gradient backpropagation. In the past years there have been many encouraging results in the application of ELM to predict protein-protein interactions (You et al., 2013), to recognize epileptic EEG models (Song \& Zhang, 2013), to estimate EEG- 
based vigilance (Shi \& Lu, 2013), to detect transmembrane beta-barrel chains (Savojardo et al., 2011), and to diagnose the thyroid disease ( $\mathrm{Li}$ et al., 2012), etc.

\section{ELM algortihm}

For $\mathrm{N}$ given training samples, an activation function $\mathrm{g}(\mathrm{x})$ and a m number of hidden neurons are taken to perform the learning of neurons as follows:

1. Random initialization of input weights Wi and bias $b_{i}$ for all $m$ number of nodes/ neurons, where $\mathrm{i}=1,2 \ldots . \mathrm{m}$;

2. Calculation of hidden layer output matrix $H$ by applying $\mathrm{X}$ over all $\mathrm{m}$ number of hidden neurons using $\mathrm{W}$ and $\mathrm{b}$ with activation function $\mathrm{g}$ as $\mathrm{g}(\mathrm{WX}+\mathrm{b})$;

3. Calculation of output weight $\beta=H^{*} T$ (1), where $H^{*}=\left(H^{T} H\right)^{-1} H^{T}$ and $\mathrm{T}=$ Output layer. Here, the objective of ELM is to minimize the training error as well as the norm of the output weights (Huang et al., 2012) in the following equation:

Minimize: $H \beta-T^{2}$ and $\beta$

where,

$H=\left[\begin{array}{ccc}g\left(W_{1} X_{1}+b_{1}\right) & \cdots & g\left(W_{m} X_{1}+b_{m}\right) \\ \cdots & \cdots & \cdots \\ g\left(W_{1} X_{N}+b_{1}\right) & \cdots & g\left(W_{m} X_{N}+b_{m}\right)\end{array}\right]_{N \times m}$

$\beta=\left[\begin{array}{c}\beta_{1}^{T} \\ \vdots \\ \beta_{m}^{T}\end{array}\right]_{m \times k} \quad T=\left[\begin{array}{c}T_{1} \\ \vdots \\ T_{N}\end{array}\right]_{N \times k}$

\subsection{OS-ELM}

When the sequential modification of ELM is based on the recursive least-square algorithm, it refers to OS-ELM. OS-ELM can learn data one-by-one or chunk-by-chunk (a block of data) with a fixed or varying chunk size. OS-ELM has proven its efficiency in several studies. For example, it is used for predicting job failure in cloud computing (Liu et al., 2017). Also, the authors in (Li et al., 2017) used the OS-ELM in gas utilization ratio prediction in blast furnaces. Furthermore, OSELM has some advantages including fast-speed and incremental learning, which demonstrate that it is suitable for target detection (Zhang et al., 2017). OS-ELM was explored in order to be used in the flood forecasting on the Neckar River, Germany (Yadav et al., 2016). The authors in (AlDahoul \& Htike, 2011) demonstrated that batch and OS-ELM algorithms are able to decode pattern activities from the visual cortex. In addition, OS-ELM was applied in watermarking (Singh et al., 2015).

\section{OS-ELM algorithm}

OS-ELM consists of two main phases: initialization and sequential learning.

Initialization phase:

- Random initialization of input weights W and bias $\mathrm{B}_{\mathrm{i}}$ for all $\mathrm{m}$ numbers of nodes/ neurons, where $\mathrm{i}=1,2 \ldots \mathrm{m}$.

- Sequential learning phase:

For the $(k+1)^{t h}$ chunk of new observation, $N_{k+1}$ denotes the number of the training data in the $(k+1)^{\text {th }}$ chunk.

(a) Calculate the partial hidden layer output matrix $\mathrm{H}_{\mathrm{k}+1}$ by using same Moore-Penrose inverse formula used in ELM.

(b) Calculate the output weight matrix $\beta^{(k+1)}$ as in equation (Liang et al., 2006) as follows:

$\beta^{(k+1)}=\beta^{(k)}+M_{k+1} H_{k+1}^{\prime}\left(T_{k+1}^{\prime}-H_{k+1} \beta^{(k)}\right.$

where

$M_{k+1}=M_{k}-M_{k} H_{k+1}^{\prime}\left(I+H_{k+1} M_{k} H_{k+1}^{\prime}\right)^{-1} H_{k+1} M_{k}$

\subsection{Proposed Algorithm of Recursive Identification based OS-ELM}

In the present method the same initializing phase of OS-ELM is maintained. For the sequential phase we follow the next algorithm:

- Sequential learning phase

The size of the chunk is 1 .

The return variable is initialized at zero

$P_{x}=$ zero $(1$, number of Inputs $)$ 
For the $(k+1)^{\text {th }}$ chunk of new observation, $N_{k+1}$ denotes the number of the training data in the $(k+1)^{t h}$ chunk.

(a) The input is composed of the new value of the database and the returned variable (the system output)

$P_{l}=\left[P_{k+1} ; P_{x}^{\prime}\right]$

where $P_{k+1}$ is the training data in the $(k+1)^{t h}$ chunk.

(b) Calculate the partial hidden layer output matrix $H_{k+1}$ by applying the same MoorePenrose inverse formula used in ELM, applying the sigmoid function

$$
H=\operatorname{Sig} \operatorname{ActFun}\left(P_{l}^{\prime}, I W^{\prime}, B_{i}\right)
$$

(c) Calculate the output weight matrix $\beta^{(k+1)}$, as in the next equation:

$\beta^{(k+1)}=\beta^{(k)}+M_{k+1} H_{k+1}^{\prime}\left(T_{k+1}^{\prime}-H_{k+1} \beta^{(k)}\right)$

Where $M_{k+1}=\operatorname{pinv}\left(M_{k}+H_{k+1}^{\prime} * H_{k+1}\right)$

(d) $P_{x}=H_{k+1} * \beta^{(k+1)}$

A diagram resuming the algorithm is presented in Figure 1.

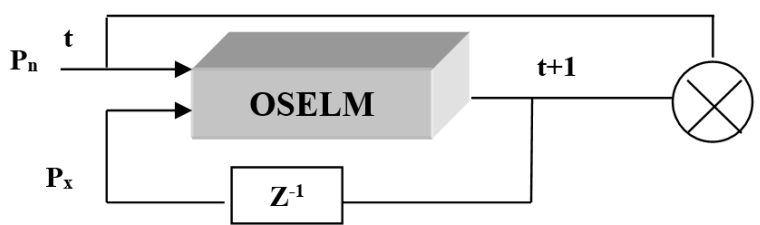

Figure 1. Diagram of recursive emotion identification system based on OS-ELM

\section{Data and Experimental Process}

One of the alternatives presented in this paper is the construction of a new database. A process is followed for analyzing EEG signals. The goal is to recognize human emotion. In this emotional experience, the same steps of the work (Blaiech et al., 2013) are maintained. However, the emotion induction protocol is changed. The difference was that the subjects do not have any idea either on the video sequence to be watched, or on the emotion stimulus. The steps of this process consist in collecting the personal information of the subjects, recording EEG signals, and then processing these signals. A protocol of emotion induction based on the EEG signals is established.

The experimental process was divided on two tasks, the first one was to induce the six emotions (neutrality, amusement, surprise, compassion, attraction, and disgust), and the second one was to predict the difficulties in video games by applying the subjects to play a selected video game having several difficulties (Table 1).

Table 1. Characteristics of used emotional EEG databases

\begin{tabular}{|c|c|c|}
\hline Databases & Emotions & Stimuli \\
\hline $\begin{array}{c}\text { DEAP } \\
\text { database }\end{array}$ & $\begin{array}{c}\text { amusement, joy, love, } \\
\text { sadness, shock }\end{array}$ & Videos \\
\hline $\begin{array}{c}\text { Our first } \\
\text { database }\end{array}$ & $\begin{array}{c}\text { neutrality, amusement, } \\
\text { surprise, compassion, } \\
\text { attraction, disgust }\end{array}$ & $\begin{array}{c}\text { Pictures, } \\
\text { videos }\end{array}$ \\
\hline $\begin{array}{c}\text { Our second } \\
\text { database }\end{array}$ & $\begin{array}{c}\text { Concentration and } \\
\text { anger }\end{array}$ & Videogames \\
\hline
\end{tabular}

\subsection{Collection of Personal Information}

The personal information of subjects is collected: each subject must fill the form of personal information containing their name, age, gender, handedness, education, alcohol/ tea/ coffee consumption, environment,...).

\subsection{EEG Recording}

The Emotiv EPOC headset is used to acquire EEG data. This headset belongs to the REGIM laboratory. It contains 14 electrodes (AF3, F7, F3, FC5, T7, P7, O1, O2, P8, T8, FC6, F4, F8 and AF4) and 2 reference electrodes. These electrodes are placed according to the standard layout of the $10-20$ system. This system refers to the spacing of the electrodes varying from 10 or $20 \%$ according to the morphology of the individual.

\subsection{Emotion Induction Protocol}

\subsubsection{First Emotional Experience}

For neutrality, neutral pictures are used (Geneva Affective PicturE Database (Dan-Glauser et al., 2011)). Amusement, surprise, compassion, attraction, and disgust are induced using video sequences. 


\subsubsection{Second Emotional Experience}

In this experience, each subject is submitted to play a selected video game named Hardest Game Ever (Anon, 2015). This game contains several steps of difficulties that can induce the concentration and anger as emotion states.

\subsection{Sample and Procedure}

\subsubsection{First Emotional Experience}

The database samples are formed from nine participants aged from 11 to 61 (five men and four women). For the first experience, each participant is submitted to watch an aleatory video sequence. Then the variation in the values of EEG signals is observed. This aleatory sequence is composed of six segments relative to emotional states : neutrality, amusement, surprise, compassion, attraction and disgust. Each segment lasts 60 seconds and represents a well-determined stimulus of emotion. At the end of each segment, an image is displayed for three seconds to distinguish the transition from one segment to another (Figure $2 \mathrm{a}$ ).

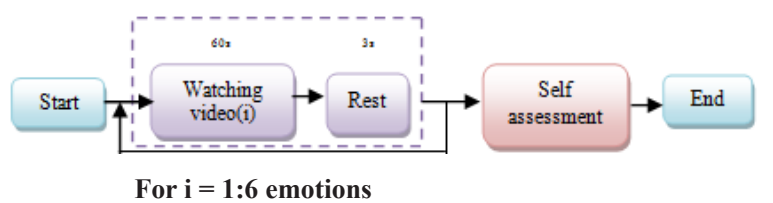

(a) First emotional experience

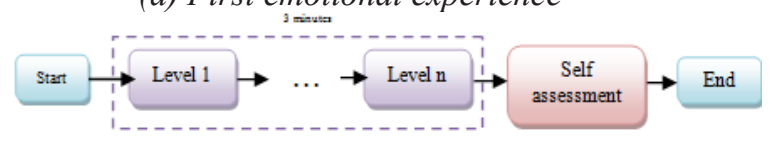

The Hardest Game Ever

(b) Second emotional experience

Figure 2. Experimental process

\subsubsection{Second Emotional Experience}

For the second experience, each subject plays the selected game during 3 minutes. The Hardest Game Ever is hard, but very entertaining as each level can be accomplished. Next level is more difficult than the previous one (Figure $2 \mathrm{~b}$ ). The latest minute of each record is retained in the samples in order to better detect the concentration and anger emotional states.

\subsection{Self-Report}

After each test, each subject is asked to provide a self-report, by describing which emotions he/she felt while watching the video sequences.

\section{Proposed Method of Identification}

The methodology used in this approach is the following (Figure 3):

- Cut the EEG data by conserving $2 / 3$ of data for learning and $1 / 3$ for testing;

- $\quad$ Apply the Independent Component Analysis (ICA) on the input EEG data;

- Extract the frequency bands (delta, theta, alpha and beta) using Fast Fourier Transform (FFT);

- Extract the 16 features previously mentioned;

- Measure the sensitivity of each feature over the bands: delta, theta, alpha and beta;

- Select the most effective/sensitive features;

- Localize the emotions in the cortex by analyzing the sensitivity rate of each EEG channel;

- Extract the results of sensitivity, specificity and accuracy of the emotional recursive identification system by applying the recursive OS-ELM algorithm;

- Retest each emotion by the recursive OS-ELM algorithm by fusing the features;

- Classify the results obtained from the identification systems, with and without considering emotion overlapping;

- Measure the performance of the system.

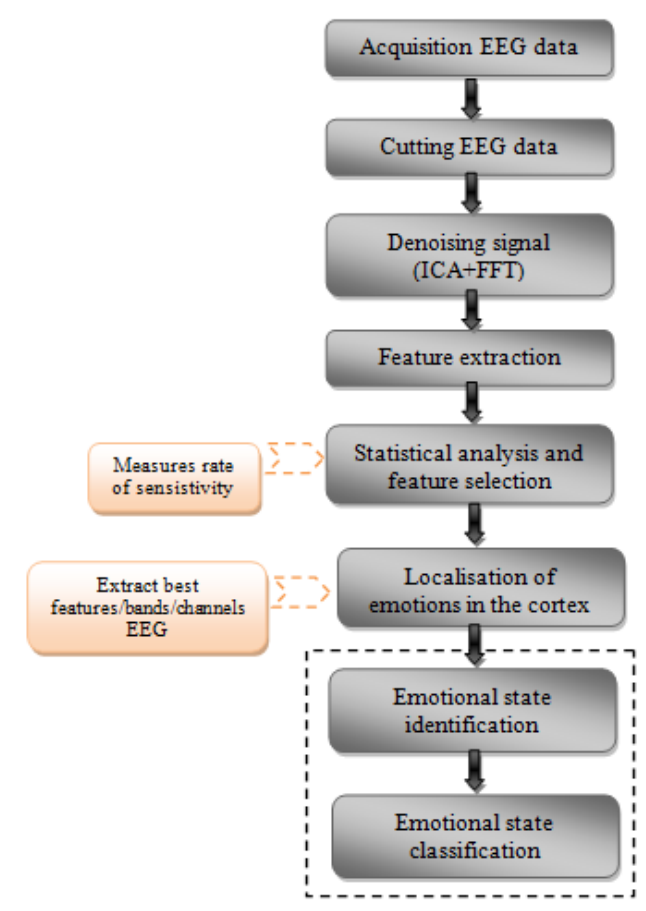

Figure 3. Emotional recognition system workflow 
This algorithm is applied on the DEAP database to validate its effectiveness. Then it is applied on a novel database constructed in this paper. The performance measures are computed as these formulas:

$$
\begin{aligned}
& \text { Accuracy }=(T P+T N) /(N+P) \\
& \text { Sensitivity }=T P / P \\
& \text { Specificity }=T N / N
\end{aligned}
$$

$N$ : Number of negative tests; $P$ : Number of positive tests; TP: Positive tests; TN: Negative tests

In this application, a set of discrete emotion models is used.

\subsection{Cutting EEG Data}

In this study, the total of EEG data for each second is 128 data (128 pieces of information). EEG recording time for each trial is 60 seconds or more for both picture stimuli and video stimuli. EEG data is cut according to the differences in the length of data for each trial. EEG data is cut to 60 seconds (7680 data) for each trial. 2/3 of these data (5120 data) are designed for learning, and 1/3 (2560 data) is designed for testing. These data are treated chunk by chunk (size: 5 seconds) as follows: 5 s are taken (640 data) of the signal. Next, this part of signal is denoised. Then the feature is extracted. After that, the data samples of the signal are incremented. Thus, 4480 data are obtained as an input to the machine learning, and 1920 data are obtained for testing. There are 14 channels in each trial and 7680 EEG data in each channel.

\subsection{Denoising Methods}

ICA is employed to denoise the EEG data (Jung et al., 2000). A bandpass filtering is applied to the EEG signal to extract the desired frequency bands. In the literature, six standard EEG bands have been defined, namely: delta, theta, alpha, mu, beta and gamma. Each of them is more prominent in different situations. According to present goals, the delta $(1-4 \mathrm{~Hz})$, theta $(4-8 \mathrm{~Hz})$, alpha $(8-13$ $\mathrm{Hz})$ and beta $(13-30 \mathrm{~Hz})$ bands will be needed, which are the most specific waves in the emotion search. The values of these waves are sought in 14 channels detected by the Emotiv EPOC headset.

\subsection{Feature Extraction}

After denoising and applying FFT on each signal, the features will be extracted from the theta, alpha and beta bands. In this study four features were extracted for each frequency band: the standard deviation (std), the average value (mean), the average value from the squared frequency band (power), and the sum of squared frequency band (energy). Indeed, the work will focus on 16 features.

\subsection{Emotional State Identification}

Recursive OS-ELM is employed for emotion identification. The properties of the system are presented in Table 2.

Table 2. Recursive OS-ELM properties

\begin{tabular}{|l|l|}
\hline Number of inputs & 2 \\
\hline Number of input neurons & 2 \\
\hline Number of hidden neurons & 5 \\
\hline Activation function & Sigmoid function \\
\hline
\end{tabular}

For each subject, there is a signal of 60 seconds. 40 seconds will be used for learning and 20 seconds for testing. The number of samples utilized for each subject in each database is presented in Table 3.

Figure 4 presents the variation in the beta parameter beta during the training of one EEG

Table 3. Number of samples for each subject

\begin{tabular}{|c|c|c|c|c|}
\hline Databases & \multicolumn{2}{|c|}{$\begin{array}{c}\text { Nbr of samples for } \\
\text { training for each subject }\end{array}$} & \multicolumn{2}{|c|}{$\begin{array}{c}\text { Nbr of samples for testing } \\
\text { for each subject }\end{array}$} \\
\hline & $\begin{array}{c}\text { Positive } \\
\text { tests }\end{array}$ & $\begin{array}{c}\text { Negative } \\
\text { tests }\end{array}$ & $\begin{array}{c}\text { Positive } \\
\text { tests }\end{array}$ & $\begin{array}{c}\text { Negative } \\
\text { tests }\end{array}$ \\
\hline $\begin{array}{c}\text { DEAP } \\
\text { database }\end{array}$ & $4480 \times 1$ & $4480 \times 4$ & $1920 \times 1$ & $1920 \times 4$ \\
\hline $\begin{array}{c}\text { Our 1st } \\
\text { database }\end{array}$ & $4480 \times 1$ & $4480 \times 5$ & $1920 \times 1$ & $1920 \times 5$ \\
\hline $\begin{array}{c}\text { Our 2nd } \\
\text { database }\end{array}$ & $4480 \times 1$ & $4480 \times 1$ & $1920 \times 1$ & $1920 \times 1$ \\
\hline
\end{tabular}

https://www.sic.ici.ro 
channel in one experiment. As it is shown, it can be noticed an increase with learning positive values and a decrease with learning negative values.

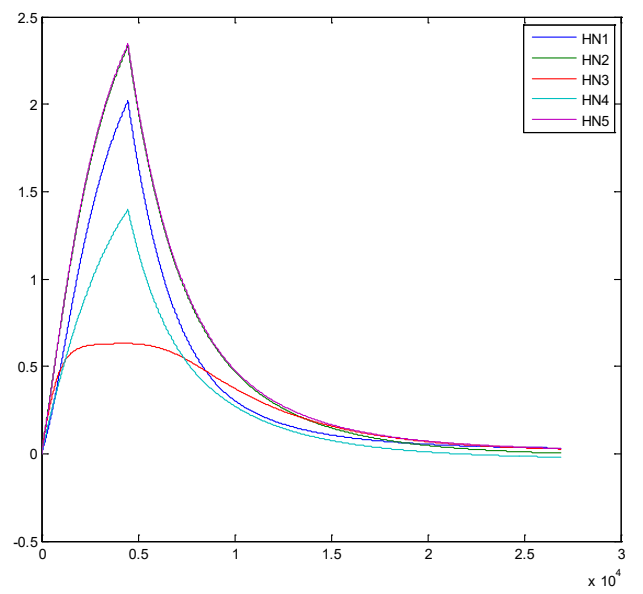

Figure 4. Variation in beta parameter (having 5 hidden neurons) during training of one EEG channel AF4 in one experiment

Figure 5 illustrates the variation in an error during the training of one EEG channel in one experiment. For both positive and negative data, the curve tends to 0 .

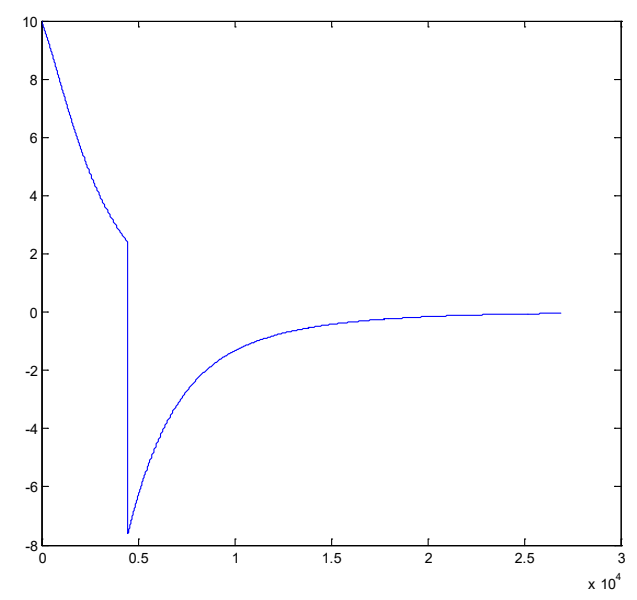

Figure 5. Variation in error during training of one EEG channel AF4 in one experiment

Figure 6 depicts the estimated value during the training of one EEG channel in one experiment. It can be seen that the signal goes up when the emotion (positive example) is elicited and is about 0 when the negative examples are elicited. In the ideal case, the outputs in positive data must be equal to 10 , and for negative data they must be equal to 0 .

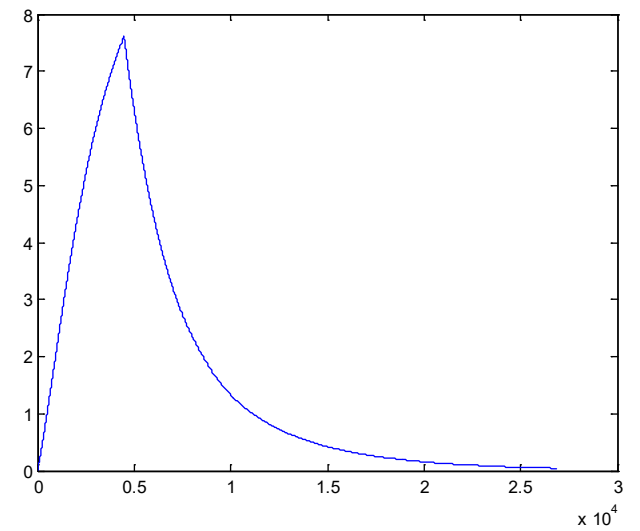

Figure 6. Estimated value during training of one EEG channel AF4 in one experiment

\subsection{Feature Selection}

For the applications performing in real time, dimensionality reduction can help to increase the speed and stability of the identification system. Hence, the frequency bands, channels and features, which have the highest rate of sensitivity are chosen. The selected ones will be used in the classification model for emotion recognition.

\subsection{Emotional State Classification}

A classifier has been set up. This classifier is composed of several subsystems of identification created in this work. The number of these subsystems is the number of emotional states taken for each experiment. They are put in parallel, and each one is constructed using the recursive OS-ELM algorithm. This classifier has two main contributions: The first is to better improve the recognition rate, and the second is to get closer to the reality of emotion. In fact, a person can have two or three emotional states at the same time with different degrees (like being in love and sad at the same time, or being amused and joyful at the same time). This shows emotion overlapping. This classifier can give the percentage of the recognition of each emotional state felt at a time (e.g. $40 \%$ sadness and $70 \%$ love).

The decision of this classifier is developed as follows:

- The output is the emotion having the maximum percentage over all emotion identification systems.

- In case that the output of the classifier is lower than $10 \%$ and the difference between the latest emotions is lower than $5 \%$, it is considered that there is an overlap of emotion and a new output of the classifier from the latest emotion is sought. 
The classifier is described in Figure 7. Emotion recognition systems are presented in Table 4 . The number of systems is relative to the number of emotions. The design of this emotion recognition system is adequate with all types of applications. It can be adapted to an emotion recognition system based on one emotion. It can also be used for a multi-emotion recognition system.

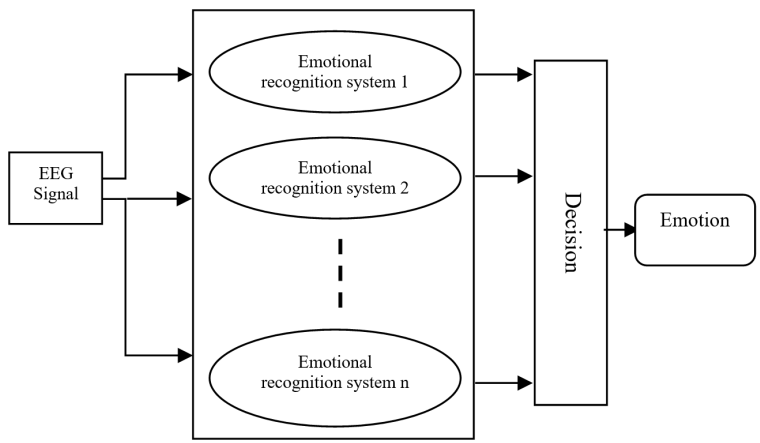

Figure 7. Model of emotion classifier

Table 4. Emotion recognition systems

\begin{tabular}{|c|c|c|}
\hline $\begin{array}{c}\text { Emotion } \\
\text { recognition } \\
\text { systems }\end{array}$ & DEAP database & Our database \\
\hline $\mathbf{1}$ & Amusement & Neutrality \\
\hline $\mathbf{2}$ & Joy & Amusement \\
\hline $\mathbf{3}$ & Love & Surprise \\
\hline $\mathbf{4}$ & Sadness & Compassion \\
\hline $\mathbf{5}$ & Shock & Attraction \\
\hline $\mathbf{6}$ & - & Disgust \\
\hline
\end{tabular}

\section{Results and Discussions}

In order to validate the efficiency of the recursive OS-ELM utilized in this study, these algorithms are firstly evaluated with the publicly available emotion dataset, the DEAP dataset. The performance of the present method is compared with the one of other methods used in the existing studies on the same emotion EEG dataset.

\subsection{Experimental Results on DEAP Database}

The DEAP database contains 32 healthy participants who participated in the experiment. 32-channel EEG signals, 4-channel EMGs, 4 Electrooculography (EOG) signals, a 2-channel Galvanic Skin Response (GSR) signal, 2 Electroretinogram (ERG) signals, the temperature in a single channel, a single channel respiration rate and 1-channel blood volume pressure were recorded. The EEG sensors were placed on the head of each participant. Then, the experimenter began recording the physiological signals, and the participant started the experiment. Each participant had to view 40 videos presented in 40 trials. At the end of each trial, the participants performed a self assessment using SAM (Bradley \& Lang, 1994), and the thumbs down / thumbs up symbols were utilized for precising liking levels (Koelstra et al., 2012). In this experiment, an emotion representation model based on five discrete emotional states from the DEAP database is adopted: amusement, joy, love, sadness and shock. We retain 21 subjects from the DEAP database.

\subsubsection{Study of Best Features and Band Frequency}

In this step there is an attempt to visualize the sensitivity rate of each feature over 21 subjects from the DEAP database. Each feature for each emotional state (amusement, joy, love, sadness and shock) is learned and tested. By analyzing the results, it can be deduced that delta band has no sensitivity in the emotional mechanism. For this, in the rest of the work, the delta band will be neglected. It can be noticed that the beta band has a better recognition rate than the other bands, except sadness where there is a balance between the alpha and beta bands. This can explain the great relationship of the alpha and beta bands with the emotional mechanism.

It can also be noticed that the energy features of the theta, alpha and beta frequency bands (the sum of squared frequency band) have the best recognition rates among the other features.

\subsubsection{Localisation of Emotion in Cortex}

Based on the sensitivity rate of each sensor from the cortex, the distributions of the EEG channels are analyzed and the best ones (the channels having the best recognition rates) are kept. These channels are used in the test phase of the recursive identification system. The distribution of channels varies between subjects and between emotional states. The average distribution of channels over all subjects through emotional states is computed. By observing the distribution of the EEG channels, it can be noticed that the frontal part of the cortex is the most responsible 
part for emotion. Except "love", which concentrates on the parietal lobe of the cortex, the occipital lobe concerns "shock". On the other hand, the alpha band is found mostly in "amusement", "joy" and "shock". Moreover, the band beta is present in most of the emotional states, except "amusement" where the rate of recognition is low. The theta band mainly concern "sadness". Table 5 summarizes the list of dominant electrodes for each emotional state, working on the average values of the sensitivity of 21 subjects.

Table 5. List of dominant electrodes for each emotional state from DEAP database

\begin{tabular}{|c|c|c|c|}
\hline Emotions & Theta & Alpha & Beta \\
\hline & \multicolumn{3}{|c|}{ Dominant electrodes } \\
\hline Amusement & - & AF3 & - \\
\hline Joy & - & F7 & AF4 \\
\hline Love & - & - & P8 \\
\hline Sadness & F3 & - & AF4 \\
\hline Shock & - & O2 & F8 \\
\hline
\end{tabular}

\subsubsection{Results of Identification System}

In this part, the results of the emotional identification system over all subjects are presented. The sensitivity, specificity and accuracy rates are measured over the five emotional states extracted from the DEAP database: amusement, joy, love, sadness and shock. The results indicate that features obtained from beta frequency bands perform better than the ones obtained from other frequency bands, which implies that beta oscillation of brain activity is more related with the processing of these three emotional states than other frequency oscillation as described in (Guntekin \& Başar, 2010). Also the fusion of features (energy of theta, alpha and beta) has a good rate of accuracy. The best results of the accuracy of our identification system for each emotion are summarized in Table 6.
Table 6. Average accuracy of recursive OS-ELM for each emotion from energy of beta band and the fusion method

\begin{tabular}{|c|c|c|c|c|c|c|}
\hline & & Amusement & Joy & Love & Sadness & Shock \\
\hline \multirow{6}{*}{$\begin{array}{c}\text { Train } \\
(\%)\end{array}$} & \multicolumn{7}{|c|}{ Beta energy } \\
\cline { 2 - 7 } & Accuracy & 69.81 & 73.06 & 71.90 & 67.66 & 70.03 \\
\cline { 2 - 7 } & Fusion of energy band \\
\cline { 2 - 7 } & Accuracy & 85.85 & 85.76 & 86.99 & 87.62 & 85.17 \\
\hline \multirow{6}{*}{$\begin{array}{c}\text { Test } \\
(\%)\end{array}$} & Beta energy \\
\cline { 2 - 7 } & Accuracy & 62.87 & 63.74 & 63.05 & 61.35 & 68.75 \\
\cline { 2 - 7 } & Accuracy & 79.63 & 77.55 & 77.36 & 78.95 & 78.64 \\
\hline
\end{tabular}

\subsubsection{Results of Classification System}

Table 7 presents the rate of emotion recognition by using a classifier composed of five classes according to the five emotional states employed. Each class refers to an emotional state. It can be noticed that the fusion method of frequency bands gives a rate of recognition higher than the one obtained by using independent frequency bands. The recognition rate in the learning and testing step is of $98.09 \%$ and of $67.61 \%$, respectively, without considering the emotion overlap in the test step. Whereas, by considering the emotion overlap, the rates are of $100 \%$ for training and of $80 \%$ for testing.

Table 7. Accuracy Rate of classification system with and without considering emotions overlap

\begin{tabular}{|c|c|c|c|}
\hline Classification & Features & $\begin{array}{c}\text { Training } \\
\text { accuracy }\end{array}$ & $\begin{array}{c}\text { Testing } \\
\text { accuracy }\end{array}$ \\
\hline \multirow{4}{*}{$\begin{array}{c}\text { Without emotion } \\
\text { overlap }\end{array}$} & Theta & 66.66 & 27.61 \\
\cline { 2 - 4 } & Alpha & 64.76 & 28.57 \\
\cline { 2 - 4 } & Beta & 65.71 & 42.85 \\
\cline { 2 - 4 } & Fusion & $\mathbf{9 8 . 0 9}$ & $\mathbf{6 7 . 6 1}$ \\
\hline \multirow{4}{*}{$\begin{array}{c}\text { Considering } \\
\text { emotions overlap }\end{array}$} & Theta & 89.52 & 55.23 \\
\cline { 2 - 4 } & Alpha & 84.76 & 55.23 \\
\cline { 2 - 4 } & Beta & 88.57 & 69.52 \\
\cline { 2 - 4 } & Fusion & $\mathbf{1 0 0}$ & $\mathbf{8 0 . 0 0}$ \\
\hline
\end{tabular}

\subsubsection{Comparison with Other Studies}

In Table 8 , the results of the present study are compared to those of some other studies working on the DEAP database.

Table 8. Comparison with some studies using DEAP Database

\begin{tabular}{|l|l|l|l|}
\hline Reference & Emotions & Method & Accuracy \\
\hline (Zhang et al., 2016) & Discrete emotions & SVM & $59.13 \%$ \\
\hline (Kumar et al., 2016) & Arousal valence model & $\begin{array}{l}\text { Bispectrum analysis, LS-SVM, ANN } \\
\text { (Linear and RBF kernels) }\end{array}$ & $64.84 \%$ \\
\hline (Atkinson \& Campos, 2015) & Arousal valence model & MRMR, SVM, genetic algorithm-SVM & $73.14 \%$ \\
\hline The present method & Discrete emotions & Recursive OS-ELM & $80.00 \%$ \\
\hline
\end{tabular}




\subsection{Experimental Results on the First} Database: Identifiying Selected Emotional States

Following the analysis of the performance of features, the feature energy considered the most efficient is used in the rest of this work. Only the theta, alpha and beta bands are kept.

\subsubsection{Neural Signatures and Stable Patterns}

To observe the neural patterns associated with emotion processing, the energy features are projected to the scalp to find temporal dynamics of frequency oscillations and stable patterns across subjects. Figure 8 depicts the average neural patterns for the neutrality, amusement, surprise, compassion, attraction and disgust emotional states. The results prove that neural signatures associated with different emotional states do exist. The lateral temporal and the frontal areas activate more for the amusement and surprise emotions than for the neutrality emotion. The energy of the prefrontal area enhances for the compassion emotion over other emotions. While the neural patterns of the surprise emotion are similar to the one of the amusement emotion, as they both have less activation in the left areas, the neural patterns of the compassion, attraction and disgust emotions have higher responses at parietal and occipital sites. As seen in the specialized literature, the authors in (Klimesh et al., 1998) prove that EEG alpha activity reflects attentional processing and beta activity reflects emotional and cognitive processes. When participants watched stimuli provoking emotion processing, and needed more concentration and more motivation, the energy of beta enhanced. The present results are consistent with the findings of the existing work (Jenke et al., 2014). Secondly, the average rates of the sensitivity of each EEG channel from the cortex are analyzed. The distribution of channels over all subjects of our database is computed. Table 9 summarizes the list of dominant sensors (if they exist) for each emotion. Through this table it can be found that "neutrality" is concentrated in the right frontal site of the cortex. The right frontal site concerns also "compassion" and "attraction". "Compassion" is founded as well concentrated in the left parietal site and the right

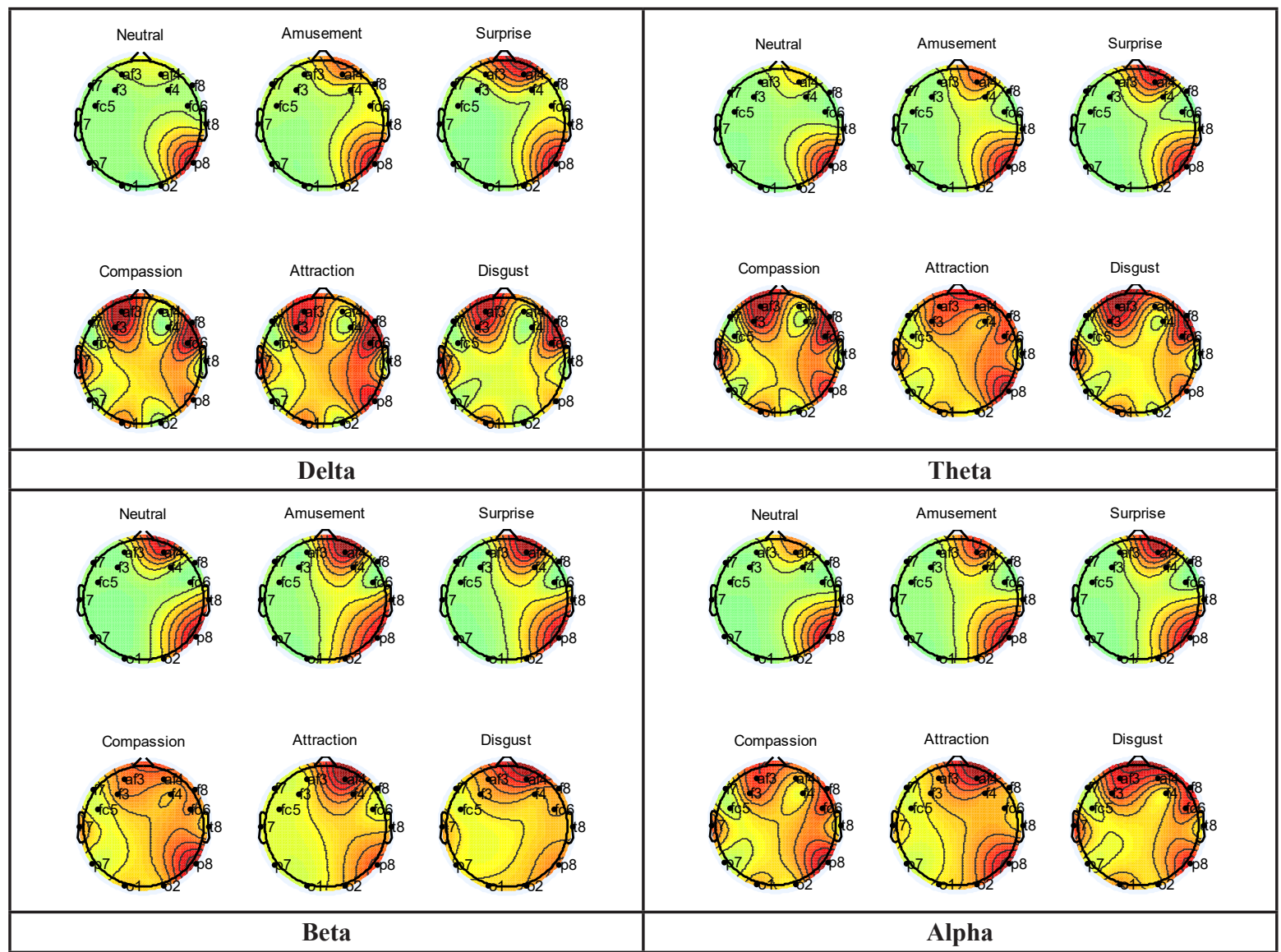

Figure 8. The average neural patterns over all subjects for different emotions from the first database 
temporal site, while "amusement" and "disgust" are concentrated in the occipital site.

Concerning the frequency bands, it can be found that the frequency theta is sensitive to "compassion" and "disgust". The alpha band can differentiate between "neutrality", "amusement", "compassion" and "attraction", while the beta band can differentiate between "neutrality" and "compassion".

Table 9. List of dominant electrodes for each emotion from the first database

\begin{tabular}{|c|c|c|c|}
\hline Emotions & Theta & Alpha & Beta \\
\hline & \multicolumn{3}{|c|}{ Dominant electrodes } \\
\hline Neutrality & - & AF4 & AF4 \\
\hline Amusement & - & O1 & - \\
\hline Surprise & - & - & - \\
\hline Compassion & T8 & AF4 & FC6, P7 \\
\hline Attraction & - & F4 & - \\
\hline Disgust & O2 & - & - \\
\hline
\end{tabular}

\subsubsection{Results of Identification System}

The rates of sensitivity, specificity and accuracy are computed over all the subjects including "neutrality", "amusement", "surprise", "compassion", "attraction" and "disgust". By observing the results during the learning and testing phases, it can be noticed that "neutrality" and "compassion" take a good rate of accuracy when the theta energy feature is used, whereas, "amusement" and "disgust" take good rates of accuracy when using the beta energy. The alpha energy feature gives good accuracy for "surprise" and "attraction". Added to that, the results fusing all the energy bands are presented. It can be noted that the fusion method gives better results than using independent frequency bands. The best rates of accuracy are illustrated in Table 10. It can be noted that the beta energy and the fusion method give the best results.

\subsubsection{Results of Classification System}

Table 11 presents the rate of emotion recognition by using a classifier composed by 6 classes. Each class refers to an emotional state. It can be noticed that the fusion method of frequency bands gives a recognition rate higher than using independent frequency bands. The recognition rate is of $87.03 \%$ in the learning step and of $40.74 \%$ in the testing step without considering emotion overlap.

Table 10. Mean of accuracy rates over subjects using beta energy and fusion method

\begin{tabular}{|c|c|c|c|c|c|c|c|c|c|c|c|c|c|}
\hline & & Neut. & & Amus. & & Surp. & & Compas. & & Attrac. & & Disgust & \\
\hline & & (1) & (2) & (1) & (2) & (1) & (2) & (1) & (2) & (1) & (2) & (1) & (2) \\
\hline \multirow{4}{*}{$\begin{array}{c}\text { Train } \\
(\%)\end{array}$} & \multicolumn{13}{|c|}{ Beta energy } \\
\hline & Accuracy & 68.40 & 16.66 & 77.10 & 20.54 & 72.51 & 21.17 & 70.50 & 16.66 & 70.64 & 16.66 & 78.66 & 45.18 \\
\hline & \multicolumn{13}{|c|}{ Fusion of features } \\
\hline & Accuracy & 83.65 & 16.66 & 84.61 & 23.55 & 85.11 & 21.27 & 79.58 & 20.25 & 82.26 & 19.62 & 87.91 & 46.34 \\
\hline \multirow{4}{*}{$\begin{array}{l}\text { Test } \\
\text { (\%) }\end{array}$} & \multicolumn{13}{|c|}{ Beta energy } \\
\hline & Accuracy & 65.06 & 16.66 & 75.81 & 16.43 & 71.95 & 20.55 & 64.44 & 16.66 & 73.05 & 16.66 & 70.33 & 43.19 \\
\hline & \multicolumn{13}{|c|}{ Fusion of features } \\
\hline & Accuracy & 75.71 & 16.66 & 78.08 & 19.33 & 81.41 & 20.87 & 75.58 & 19.25 & 79.79 & 19.25 & 74.98 & 44.71 \\
\hline
\end{tabular}

(1): Our method, (2):SVM

Table 11. Accuracy rate of classification system with and without considering emotion overlap

\begin{tabular}{|c|c|c|c|}
\hline & Features & Train Accuracy & Test Accuracy \\
\hline \multirow{4}{*}{$\begin{array}{c}\text { Without } \\
\text { emotion overlap }\end{array}$} & Theta & 35.18 & 16.66 \\
\cline { 2 - 4 } & Alpha & 40.74 & 20.37 \\
\cline { 2 - 4 } & Beta & 46.29 & 18.51 \\
\cline { 2 - 4 } & Fusion & $\mathbf{8 7 . 0 3}$ & $\mathbf{4 0 . 7 4}$ \\
\hline \multirow{4}{*}{$\begin{array}{c}\text { Considering } \\
\text { emotions } \\
\text { overlap }\end{array}$} & Theta & 81.48 & 55.55 \\
\cline { 2 - 4 } & Alpha & 77.77 & 50 \\
\cline { 2 - 4 } & Beta & 81.48 & 68.51 \\
\cline { 2 - 4 } & Fusion & $\mathbf{1 0 0}$ & $\mathbf{7 4 . 0 7}$ \\
\hline
\end{tabular}


When considering the emotion overlap, the rate is of $100 \%$ for training and of $74.07 \%$ for testing.

\subsection{Experimental Results on the Second Database: Predicting Difficulties in Video Games}

\subsubsection{Neural Signatures and Stable Patterns}

Figure 9 depicts the average neural patterns for the neutral emotion and the state describing the difficulties encountered while playing video game. The results prove that neural signatures associated with these two states do exist. While playing the game, the energy of the prefrontal area enhances. Also the temporal and occipital sites of the right area activate more. The present results are congruent to those of the existing studies (Berntson et al., 2011) which evocate that negative emotions are associated more with the right rather than to the left hemispheric activity. Secondly, the average rates of the sensitivity of each EEG channel from the cortex are computed. The results of the distribution of channels over all the subjects of this database are presented in Table 12. By observing the dominant sensors over emotions, it can be noticed that "neutrality" concentrates in the frontal site in the theta band and the right frontal site in the beta band. Concerning the emotions generated by the difficulties encountered in the video games, which are the concentration and anger states, it can be noticed that these states are found in the right fontal site of the cortex in the theta, alpha and beta bands.

Table 12. List of dominant electrodes for each emotion from the second database

\begin{tabular}{|c|c|c|c|}
\hline Emotions & Theta & Alpha & Beta \\
\hline \multicolumn{4}{|c|}{ Dominant electrodes } \\
\hline Neutrality & FC5 & - & AF4 \\
\hline Concentration and Anger & AF4,FC6 & AF4,FC6 & AF4 \\
\hline
\end{tabular}

\subsubsection{Results of Identification System}

The rates of sensitivity, specificity and accuracy are computed over all the subjects including "neutrality" and the emotion state depicting the difficulties encountered in the video games which is "concentration with anger". These figures show that the energy features of the beta band and of the fusion method take the best rates of sensitivity, specificity and accuracy (Table 13).

\subsubsection{Results of Classification System}

Table 14 illustrates the results obtained from the classifier composed by two classes referring to the two emotion states "neutrality" and "concentration with anger". The feature energy of beta has the best recognition rates of $94.44 \%$ in the training phase and of $61.11 \%$ in the testing phase.

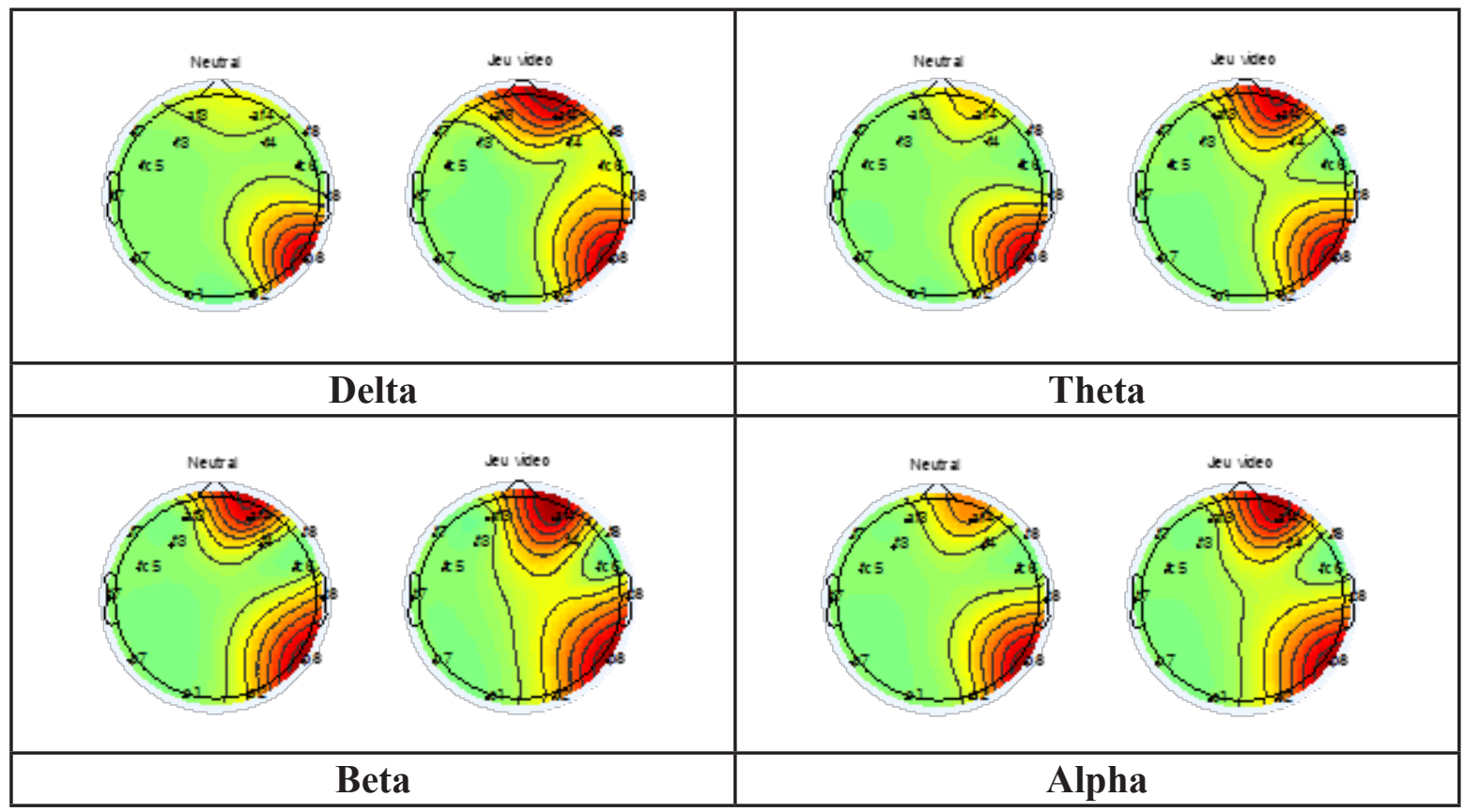

Figure 9. The average neural patterns over all subjects for different emotions from the second database (Predicting difficulties in video game) 
Table 13. Average accuracy of recursive OS-ELM for each emotion from energy of beta band and our fusion method

\begin{tabular}{|c|c|c|c|c|c|}
\hline & & Neutral & & entration and & \\
\hline \multirow{5}{*}{$\begin{array}{c}\text { Train } \\
(\%)\end{array}$} & & Our method & SVM & Our method & SVM \\
\hline & \multicolumn{5}{|c|}{ Beta energy } \\
\hline & Accuracy & 72.50 & 58.70 & 61.45 & 51.55 \\
\hline & \multicolumn{5}{|c|}{ Fusion of features } \\
\hline & Accuracy & 81.43 & 60.41 & 70.57 & 64.76 \\
\hline \multirow{4}{*}{$\begin{array}{l}\text { Test } \\
(\%)\end{array}$} & \multicolumn{5}{|c|}{ Beta energy } \\
\hline & Accuracy & 52.15 & 52.90 & 52.83 & 48.25 \\
\hline & \multicolumn{5}{|c|}{ Fusion of features } \\
\hline & Accuracy & 50.88 & 51.41 & 51.50 & 55.27 \\
\hline
\end{tabular}

Table 14. Accuracy Rate of classification system

\begin{tabular}{|c|c|c|c|}
\hline & Features & Train Accuracy & Test Accuracy \\
\hline \multirow{4}{*}{$\begin{array}{c}\text { Without emotion } \\
\text { overlap }\end{array}$} & Theta & 83.33 & 38.88 \\
\cline { 2 - 4 } & Alpha & 72.22 & 44.44 \\
\cline { 2 - 4 } & Beta & $\mathbf{9 4 . 4 4}$ & $\mathbf{6 1 . 1 1}$ \\
\cline { 2 - 4 } & Fusion & 100 & 44.44 \\
\hline
\end{tabular}

\subsection{Discussion}

This novel algorithm of identification, based on the principle of recursivity and OS-ELM, has better results than those of the algorithms from other studies using the DEAP database. By testing the DEAP database, the results are analyzed and the best features are selected. The energy features have the best rates of accuracy in comparison with the mean, the standard deviation and the power. It can be deduced that the beta band is the most responsible for emotion identification. Firstly, a new database is created to incite new emotions that do not exist in the DEAP database such as "disgust", "surprise" and "compassion". Secondly, subjects having our culture and from our environment are used. Thirdly, a hard video game that contain several stages is chosen, to identify the difficulties encountered when playing. Then the concentration and anger emotion states that the subjects feel while playing video games are detected. From the two emotional experiences it can be deduced that the beta band is mostly sensitive to emotional states, and that the delta band has no sensitivity to emotional states. The present fusion method has the best results of emotion identification, as well as the best classification rates, while the feature energy of beta has the best rates of accuracy in predicting difficulties in video games. Furthermore, the classifier improves the recognition rates. The SVM method was applied in the present samples (Schölkopf et al. 2002) (Tables 10 and 13). By comparing the recursive identification method based on OS ELM, to the SVM method, it was found that the present method has the best rates of identification.

\section{Conclusion}

In this paper a novel algorithm based on OS-ELM and recursive identification has been proposed. This algorithm has been validated on the publicly DEAP database. Two new databases have been constructed. In fact, two emotional experiences using the emotiv epoc headset have been accomplished. In the first step, six emotional states have been induced by utilizing image and video stimuli. In the second step, we incited the subjects to play a hard video game. The goal was to predict the difficulties encountered when playing and to identify the concentration and anger emotional states. The present learning algorithm has been applied on two new databases and the results of this experience have been analyzed by observing the localization of these emotional states in the cortex. Some parts of the lobe cranial responsible of some emotional states have been noticed. This work has been divided in two parts: identification and classification. In the 
identification part, each emotion (identified or not) has been treated independently. For the classification part, the performance has been measured by computing the highest recognition rates of each identification system. Finally, the present method has been compared to the SVM method and it has been proved that the method employed in this paper has had the best rates of identification. This system has achieved good recognition rates: $80 \%$ when using the DEAP

\section{REFERENCES}

AlDahoul, N., \& Htike, Z. (2015). Online Sequential Extreme Learning Machine Based Functional Magnetic Resonance Imaging Decoder, Advanced Science Letters, 21(11), 3489-3493.

Anon (2015). Hardest Game Ever. Available at: <https://www.hardestgameever.net/>, last accessed: 2018.

Atkinson, J. \& Campos, D. (2015). Improving BCIbased emotion recognition by combining EEG feature selection and kernel classifiers, Expert Systems with Appications, 47, 35-41. DOI: 10.1016/j. eswa.2015.10.049.

Balconi, M., Grippa, E. \& Vanutelli, M. E. (2015). What hemodynamic (fNIRS), electrophysiological (EEG) and autonomic integrated measures can tell us about emotional processing, Brain and Cognition, 95, 67-76. DOI: 10.1016/j.bandc.2015.02.001

Barrett, L. F. \& Russell, J. A. (1998). Independence and bipolarity in the structure of current affect, Journal of Personality and Social Psychology, 74(4), 967-984.

Berntson, G. G., Norman, G. J. \& Cacioppo, J. T. (2011). Comment: laterality and evaluative bivalence: a neuroevolutionary perspective, Emotion Review, 3, 344-346.

Blaiech, H., Neji, M., Wali, A. \& Alimi, M. A. (2013). Emotion Recognition by Analysis of EEG Signals. In The 13th International conference on Hybrid Intelligent Systems, HIS 2013, December 04-06, 2013, Hammamet-Tunisia (pp. 312-318). DOI: 10.1109/ HIS.2013.6920451

Bradley, M. M. \& Lang, P. J. (1994). Measuring emotion: the selfassessment manikin and the semantic differential, Journal of Behavior Therapy and Experimental Psychiatry, 25(1), 49-59.

Dan-Glauser, E. S. \& Scherer, K. R. (2011). The Geneva affective picture database (GAPED): a new 730-picture database focusing on valence and normative significance, Behavior Research Methods, 43(2), 468-477. database, $74.07 \%$ when utilizing this first newly database, and $61.11 \%$ when predicting difficulties in video games. a perspective of this work, the number of emotional states can be enlarged by treating other more complex ones. The human emotion of other cultures cannot be treated yet. In a next step, this algorithm will be implemented in a Field Programmable Gate Arrays (FPGA) platform in order to gain the time of execution.

Güntekin, B. \& Basar, E. (2010). Event-related beta oscillations are affected by emotional eliciting stimuli, Neuroscience letters, 483(3), 173-178.

Huang, G. B., Zhou, H., Ding, X. \& Zhang, R. (2012). Extreme Learning Machine for Regression and Multiclass Classification, IEEE Transactions on Systems, Man, And Cybernetics - Part B: Cybernetics, 42(2), 513-529.

Jenke, R., Peer, A. \& Buss, M. (2014). Feature extraction and selection for emotion recognition from EEG, IEEE Transactions on Affective Computing, 5(3), 327-339.

Jung, T.-P., Makeig, S., Humphries, C., Lee, T.-W., McKeown, M., Iragui, V. \& Sejnowski, T. (2000). Removing electroencephalographic artifacts by blind source separation, Psychophysiology, 37(2), 163-178.

Klimesch, W., Doppelmayr, M., Russegger, H., Pachinger, T. \& Schwaiger, J. (1998). Induced alpha band power changes in the human EEG and attention, Neuroscience letters, 244(2), 73-76.

Koelstra S., Muhl, C., Soleymani, M., Lee, J. S., Yazdani, A., Ebrahimi, T, Pun, T., Nijholt, A. \& Patras, I. (2012). DEAP: a database for emotion analysis; using physiological signals, IEEE Transactions Affective Computing, 3(1), 18- 31. DOI: 10.1109/T-AFFC.2011.15

Kumar, N., Khaund, K. \& Hazarika, S. M. (2016). Bispectral Analysis of EEG for Emotion Recognition, Procedia Computer Science, 84, 31-35. DOI: 10.1016/j.procs.2016.04.062.

Li, L. N., Ouyang, J. H., Chen, H. L \&, Liu, D.Y. (2012). A computer aided diagnosis system for thyroid disease using extreme learning machine, Journal of Medical Systems, 3(5), 3327-3337.

Li. S. \& Deng W. (2018). Deep Facial Expression Recognition: A Survey, ArXiv, abs/1804.08348.

Li, Y., Zhang, S., Yin, Y., Xiao, W. \& Zhang, J. (2017). A Novel Online Sequential Extreme Learning

https://www.sic.ici.ro 
Machine for Gas Utilization Ratio Prediction in Blast Furnaces, Sensors, 17(8), 1847.

Liang, N., Huang, G. \& Saratchandran, P. \& Sundararajan, N. (2006). A Fast and Accurate Online Sequential Learning Algorithm for Feedforward Networks, IEEE Transactions on Neural Networks, 17(6), 1411-142. DOI: 10.1109/TNN.2006.880583

Liu, C., Han, J., Shang, Y., Liu, C., Cheng, B. \& Chen, J. (2017). Predicting of Job Failure in Compute Cloud Based on Online Extreme Learning Machine: A Comparative Study, IEEE Access, 5, 9359-9368. DOI: 10.1109/ACCESS.2017.2706740

Ng, H.-W. \& Nguyen, D., Vonikakis, V. \& Winkler, S. (2015). Deep Learning for Emotion Recognition on Small Datasets Using Transfer Learning. In ACM International Conference on Multimodal Interaction, Seattle, Washington, USA (pp. 443-449). DOI: $10.1145 / 2818346.2830593$

Palaniswamy, S. \& and Tripathi, S. (2018). Emotion Recognition from Facial Expressions using Images with Pose, Illumination and Age Variation for HumanComputer/Robot Interaction, Journal of ICT Research and Applications, 12(1), 14-34.

Petrantonakis, P. C. \& Hadjileontiadis, L. J. (2009). EEG-Based Emotion Recognition Using Hybrid Filtering and Higher Order Crossings. In: 2009 3rd International Conference on Affective Computing and Intelligent Interaction and Workshops, 10-12 September 2009, Amsterdam (pp. 1-6). DOI: 10.1109/ ACII.2009.5349513

Picard, R. W. (2000). Affective Computing. Cambridge, MA: The MIT Press.

Savojardo, C., Fariselli, P. \& Casadio, R. (2011). Improving the detection of transmembrane betabarrel chains with n-to-1 extreme learning machines, Bioinformatics, 27(22), 3123-3128.

Schölkopf, S. (2002). Learning with Kernels: Support Vector Machines, Regularization, Optimization and Beyond. MIT Press.
Shi, L. C. \& Lu, B. L. (2013). EEG-based vigilance estimation using extreme learning machines, Neurocomputing, 102, 135-143.

Singh, R.P., Dabas, N., Chaudhary, V., Nagendra (2015) Online Sequential Extreme Learning Machine for Watermarking. In: Cao, J., Mao, K., Cambria, E., Man, Z. \& Toh, K. A. (eds.), Proceedings of ELM2014 Volume 2. Proceedings in Adaptation, Learning and Optimization, vol 4, 115-124. Springer, Cham. DOI: 10.1007/978-3-319-14066-7_12

Song, Y. D. \& Zhang, J. X. (2013). Automatic recognition of epileptic EEG patterns via extreme learning machine and multiresolution feature extraction, Expert Systems with Applications, 40(14), 5477-5489.

Yadav, B., Ch, S., Mathur, S. \& Adamowski, J. (2016). Discharge forecasting using an Online Sequential Extreme Learning Machine (OS-ELM) model: A case study in Neckar River, Germany, Measurement, 92, 433-445.

Yang, D., Alsadoon, A., Prasad, P.W.C., Singh, A. K. \& Elchouemi, A. (2018). An Emotion Recognition Model Based on Facial Recognition in Virtual Learning Environment, Procedia Computer Science, 125, 2-10.

You, Z. H., Lei, Y. K., Zhu, L., Xia, J. F. \& Wang, B. (2013). Prediction of protein-protein interactions from amino acid sequences with ensemble extreme learning machines and principal component analysis, BMC Bioinformatics, 14, 1-11. Article number: S10.

Zhang, J., Chen, M., Zhao, S., Hu, S., Shi, Z. \& Cao, Y. (2016). ReliefF-Based EEG Sensor Selection Methods for Emotion Recognition, Sensors, 16(10), 1558. DOI: $10.3390 / \mathrm{s} 16101558$

Zhang, J., Feng, L. \& Yu, L. (2017). A novel target tracking method based on OS-ELM, Multidimensional Systems and Signal Processing, 28(3), 1091-1108. 